\title{
Global beam shaping with nonuniformly polarized beams: a proposal
}

\author{
V. Ramírez-Sánchez and G. Piquero
}

\begin{abstract}
A procedure for global beam shaping by modifying some global spatial parameters characteristic of the beam is proposed. This method is based on the generation of a nonuniformly polarized beam using a Mach-Zehnder system with two suitably shaped intensity transmittances and orthogonal linear polarizers. The changes in beam quality and kurtosis parameters after a linear polarizer placed at the output of the system are investigated. (C) 2006 Optical Society of America

OCIS codes: $260.5430,140.3300$.
\end{abstract}

\section{Introduction}

As is well known, optical devices that modify the irradiance distributions of laser beams are useful in many applications, such as sources of heat for drilling, welding, and surface treatment. Laser fusion experiments, information recording, or optical data processing are other applications in which an appropriate transversal profile is required. A large number of methods for beam shaping have been proposed and implemented. They are based on different phenomena, such as refraction, ${ }^{1}$ reflection of beams over prescribed surfaces, ${ }^{2-4}$ diffraction, ${ }^{5}$ or absorption. ${ }^{6}$ The use of filters or apodization methods are additional approaches to beam shaping. ${ }^{7,8}$ All the techniques mentioned above are based on the scalar theory of light without taking into account its vectorial character. There are few papers that use the phenomenon of polarization for beam shaping. For example, Simmons et al. ${ }^{9}$ designed a device based on the Pockels and Faraday effects in order to profile a plane-polarized, collimated, and monochromatic wave. Birefringent lenses are included in laser resonators to obtain predetermined profiles. ${ }^{10,11}$ All the above methods are focused on modifying the distribution of the irradiance at each point of the transverse section of the beam.

On the other hand, the spatial structure of laser beams can be characterized by means of some glob-

The authors are with the Departamento de Óptica, Facultad de Ciencias Físicas, Universidad Compultense, 28040 Madrid, Spain. G. Piquero's e-mail address is piquero@fis.ucm.es.

Received 9 June 2006; revised 1 August 2006; accepted 3 August 2006; posted 7 August 2006 (Doc. ID 71714).

0003-6935/06/358902-05\$15.00/0

(C) 2006 Optical Society of America al parameters based on the so-called intensity moments. ${ }^{12-16}$ The beam quality parameter ${ }^{15}$ and kurtosis $^{17}$ are two of them. For some particular applications adequate values of these parameters are required, and it is necessary to design optical systems that modify the global characteristics of the beam. In this sense we will say we are doing a global beam shaping. Some optical elements have been proposed to change both parameters, such as binary phase plates, ${ }^{18}$ quartic phase plates, ${ }^{19}$ aberrated lenses, ${ }^{20}$ and axicons, ${ }^{21}$ among others.

In the present paper we propose what we believe to be a new procedure for changing the global characteristics of beams taking the vectorial nature of light into account. The method is based on the synthesis of totally and nonuniformly polarized beams with a Mach-Zehnder interferometer. Two different amplitude filters and two orthogonal polarizers placed at each arm of the interferometer cause a nonuniform change in the state of polarization over the transversal section of the beam. By rotating a linear polarizer at the output of the interferometer, we modify the irradiance of the beam and its global characteristics. The changes in beam quality and the kurtosis parameters are investigated.

The paper is structured as follows. After the introduction in Section 1, the definitions and some properties of the beam quality and kurtosis parameters are presented in Section 2. The procedure used for global beam shaping by generating a totally nonuniformly polarized beam is explained in Section 3. Finally, the conclusions are given in Section 4.

\section{Formalism and Definitions}

Let us start with a monochromatic plane wave propagating along the $z$ axis, written in terms of its $s$ and 
$p$ components as follows:

$$
\mathbf{E}(x ; z)=\left[E_{s}(x ; z), E_{p}(x ; z)\right] .
$$

Note that for the sake of simplicity, we shall refer to a two-dimensional case in which one of the transverse coordinates can be neglected. We define the moments of a partially polarized beam using ${ }^{15}$

$$
\begin{aligned}
\langle\alpha \beta\rangle_{j}= & \frac{1}{I_{j}} \iiint \alpha \beta \overline{E_{j}^{\dagger}(x+s / 2, z) E_{j}(x-s / 2, z)} \\
& \times \exp (\mathrm{i} k s u) \mathrm{d} s \mathrm{~d} x \mathrm{~d} u,
\end{aligned}
$$

where the symbol $\dagger$ denotes the adjoint and the overbar represents an ensemble average. In Eq. (2) $x$ is the spatial coordinate; $u$ represents an angle of propagation (without taking the evanescent waves into account); $\alpha, \beta=x, u$; and the subscript $j$ refers to the $s$ or $p$ components of the field.

In terms of these measurable moments the expression of the beam quality parameter in the waist plane is given by ${ }^{22}$

$$
Q=\frac{I_{s}^{2}}{I^{2}} Q_{s}+\frac{I_{p}^{2}}{I^{2}} Q_{p}+\frac{I_{s} I_{p}}{I^{2}} Q_{s p},
$$

with

$$
\begin{gathered}
Q=\left\langle x^{2}\right\rangle\left\langle u^{2}\right\rangle, \\
Q_{s}=\left\langle x^{2}\right\rangle_{s}\left\langle u^{2}\right\rangle_{s}, \\
Q_{p}=\left\langle x^{2}\right\rangle_{p}\left\langle u^{2}\right\rangle_{p}, \\
Q_{s p}=\left\langle x^{2}\right\rangle_{s}\left\langle u^{2}\right\rangle_{p}+\left\langle x^{2}\right\rangle_{p}\left\langle u^{2}\right\rangle_{s},
\end{gathered}
$$

where $I_{s}$ and $I_{p}$ are the powers associated with the $s$ and $p$ components, respectively, and $I=I_{s}+I_{p}$. Usually, $\left\langle x^{2}\right\rangle$ and $\left\langle u^{2}\right\rangle$ are connected with the beam width and the far-field divergence of each field component. For simplicity, we are considering $\langle x\rangle=\langle u\rangle=0$. The minimum value of $Q$ is reached by totally and uniformly Gaussian beams and takes the value $Q_{g}=1 / 4 k^{2}$, with $k=2 \pi / \lambda$ the wavenumber and $\lambda$ the wavelength of the beam. Note that $Q$ is related to the standard beam propagation factor $M^{2}$ through the expression $\left(M^{2}\right)^{2}=k^{2} Q$. The beam quality factor, $Q$, gives us an idea about the focusing and collimation capabilities of a laser beam. These characteristics are related to the high focusability (small beam width) along with low beam divergence at the far field, which are the required conditions in numerous laser applications. The beam quality parameter is invariant upon propagation through the $A B C D$ optical systems.
On the other hand, another useful parameter is the kurtosis parameter defined in terms of higher-order averages of the beam as ${ }^{17}$

$$
K=\frac{\left\langle x^{4}\right\rangle}{\left\langle x^{2}\right\rangle^{2}}
$$

with

$$
\begin{aligned}
& \left\langle x^{4}\right\rangle=\frac{I_{s}}{I}\left\langle x^{4}\right\rangle_{s}+\frac{I_{p}}{I}\left\langle x^{4}\right\rangle_{p}, \\
& \left\langle x^{2}\right\rangle=\frac{I_{s}}{I}\left\langle x^{2}\right\rangle_{s}+\frac{I_{p}}{I}\left\langle x^{2}\right\rangle_{p} .
\end{aligned}
$$

Taking this definition into account, the profile of any laser beam can be classified as leptokurtic, mesokurtic, or platykurtic, depending on whether $K$ is higher than, equal to, or lower than 3 , which is the kurtosis value of a pure Gaussian beam $\left(K_{g}\right)$. Leptokurtic and platykurtic profiles imply sharper and flatter beams, respectively, than in the Gaussian case.

\section{Global Beam Shaping}

The totally and nonuniformly polarized beam would be generated using a Mach-Zehnder interferometer. Two orthogonal linear polarizers and two different amplitude filters are placed at each arm. In our particular case we have chosen the following transmittances:

$$
\begin{aligned}
& t_{m}(x)=\exp \left[-\frac{1}{2}(a x)^{2 m}\right] \\
& t_{n}(x)=\exp \left[-\frac{1}{2}(b x)^{2 n}\right],
\end{aligned}
$$

where $a$ and $b$ are positive constants related to the aperture widths.

Within the framework of the Jones matrix treatment, to determine the field amplitude at the output plane of the Mach-Zehnder system (MZS), we will make use of the paraxial approximation, which is consistent with the intensity moment treatment. The Jones vector at the output of the MZS takes the form

$$
\mathbf{E}^{(o)}(x)=\left(\begin{array}{l}
t_{m}(x) E_{s}{ }^{(i)}(x) \\
t_{n}(x) E_{p}{ }^{(i)}(x)
\end{array}\right),
$$

where the superscripts $(o)$ and $(i)$ refer to the output and input beams, respectively. From Eq. (13) it can be seen that $\mathbf{E}^{(o)}$ represents a totally and nonuniformly polarized beam.

By using Eqs. (2)-(13) the following analytical expressions for the beam quality and kurtosis parameter of the field $\mathbf{E}^{(o)}$ are derived: 


$$
\begin{aligned}
& Q=\frac{\left[\frac{a^{-3}}{2 m} \Gamma\left(\frac{3}{2 m}\right)+\frac{b^{-3}}{2 n} \Gamma\left(\frac{3}{2 n}\right)\right]\left[\frac{m a}{2} \Gamma\left(2-\frac{1}{2 m}\right)+\frac{n b}{2} \Gamma\left(2-\frac{1}{2 n}\right)\right]}{k^{2}\left[\frac{a^{-1}}{2 m} \Gamma\left(\frac{1}{2 m}\right)+\frac{b^{-1}}{2 n} \Gamma\left(\frac{1}{2 n}\right)\right]^{2}}, \\
& K=\frac{\left[\frac{a^{-5}}{2 m} \Gamma\left(\frac{5}{2 m}\right)+\frac{b^{-5}}{2 n} \Gamma\left(\frac{5}{2 n}\right)\right]\left[\frac{a^{-1}}{2 m} \Gamma\left(\frac{1}{2 m}\right)+\frac{b^{-1}}{2 n} \Gamma\left(\frac{1}{2 n}\right)\right]}{\left[\frac{a^{-3}}{2 m} \Gamma\left(\frac{3}{2 m}\right)+\frac{b^{-3}}{2 n} \Gamma\left(\frac{3}{2 n}\right)\right]^{2}},
\end{aligned}
$$

where $\Gamma$ is the gamma function. We can see that $Q$ and $K$ depend on the transmittance parameters, $m, n$, $a$, and $b$. From Eq. (14) we find that $Q$ reaches a minimum value when $m=n$ and $a=b$. In such a case we have a uniformly totally polarized beam.

To modify the parameters given by Eqs. (14) and (15) a linear polarizer is placed at the output plane of the MZS. In this way, by rotating this polarizer we can perform a global beam shaping. The Jones vector at the output of the polarizer, whose transmission axis forms an angle $\theta$ with the $x$ axis, is

$$
\begin{aligned}
\mathbf{E}^{\prime(o)}(x)= & {\left[E_{s}{ }^{(i)}(x) t_{m}(x) \cos \theta+E_{p}{ }^{(i)}(x) t_{n}(x) \sin \theta\right] } \\
& \times\left(\begin{array}{l}
\cos \theta \\
\sin \theta
\end{array}\right),
\end{aligned}
$$

where the prime indicates the parameters after the polarizer. The field $\mathbf{E}^{\prime(o)}(x)$ is totally and uniformly polarized. Note that for material processing the polarization of light is another feature to take into account. ${ }^{23}$

For the particular cases of $\theta=0^{\circ}$ and $90^{\circ}$, we find that the beam quality and kurtosis parameters after the polarizer are independent of the widths $a$ and $b$.

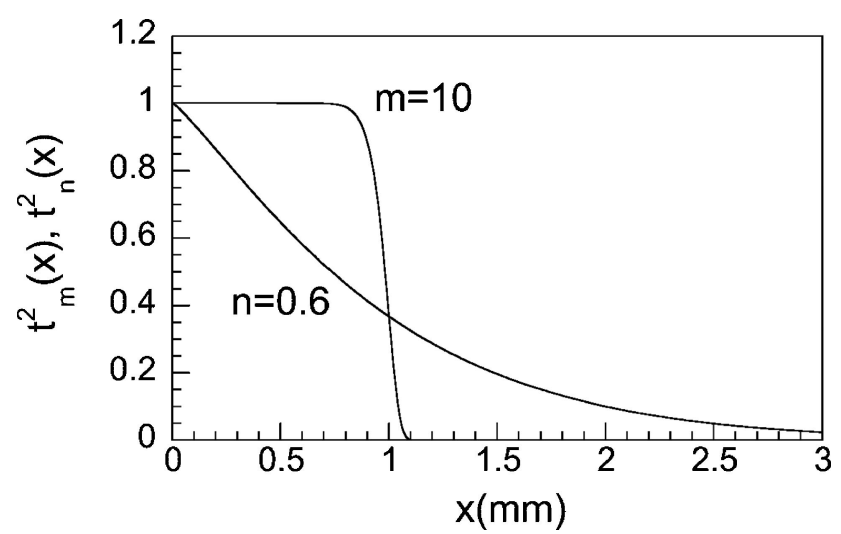

Fig. 1. Squared profiles of the transmittances, $t_{m}{ }^{2}(x)$ and $t_{n}{ }^{2}(x)$, for $a=b=1 \mathrm{~mm}^{-1}$ and $m=10$ and $n=0.6$.
They take the following simple analytical forms:

$$
\begin{aligned}
& Q_{l}{ }^{\prime}=\frac{l^{2}}{k^{2}} \frac{\Gamma(3 / 2 l) \Gamma(2-1 / 2 l)}{\Gamma(1 / 2 l)^{2}}, \\
& K_{l}{ }^{\prime}=\frac{\Gamma(5 / 2 l) \Gamma(1 / 2 l)}{\Gamma(3 / 2 l)^{2}},
\end{aligned}
$$

with $l$ equal to $m$ for $\theta=0^{\circ}$ and equal to $n$ for $\theta=$ $90^{\circ}$. For other angles, $Q^{\prime}$ and $K^{\prime}$ depend on the transmittance parameters.

To analyze the variation of the beam quality and kurtosis parameters when the polarizer rotates, we use two amplitude filters with $m=10, n=0.6$, and $a=b=1 \mathrm{~mm}^{-1}$ as an example. Squared profiles of these transmittances have been plotted in Fig. 1. The beam intensity distribution coincides with the profiles of Fig. 1 when the transmission axis of the polarizer is $0^{\circ}$ and $90^{\circ}$, respectively. On the other hand,

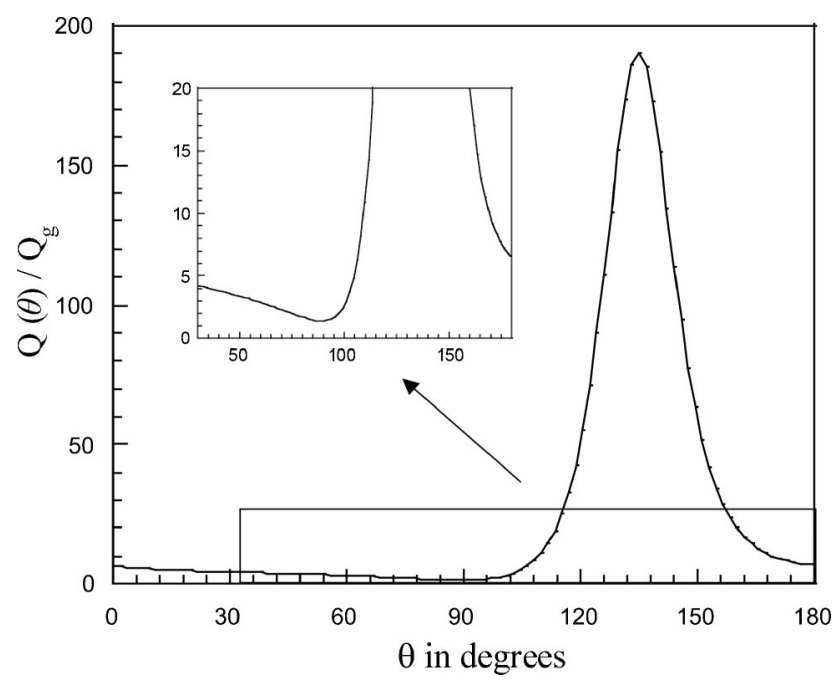

Fig. 2. Beam quality parameter at the output of the polarizer normalized to the beam quality of a Gaussian beam with the same wavelength, $Q^{\prime} / Q_{g}$, versus the angle of the polarizer $\theta$. 


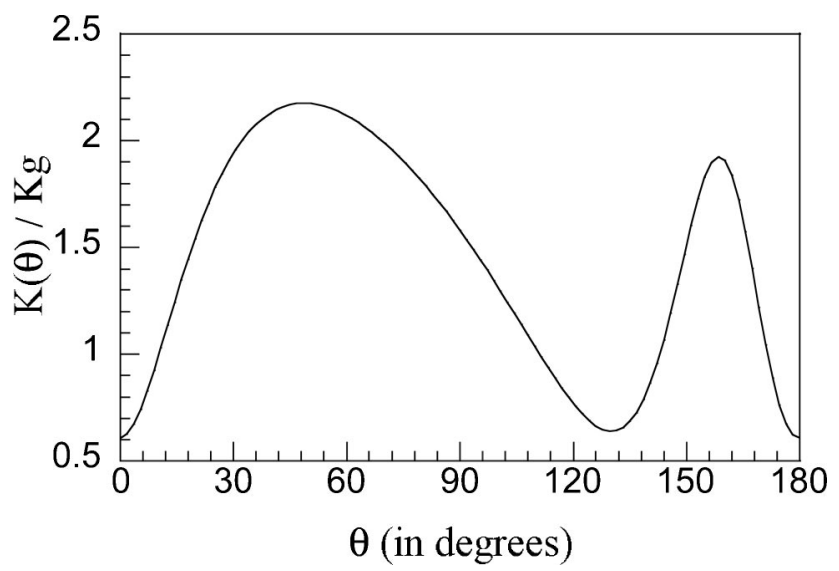

Fig. 3. Kurtosis parameter at the output of the polarizer normalized to the kurtosis of a Gaussian beam with the same wavelength, $K^{\prime} / K_{g}$, versus the angle of the polarizer $\theta$.

from Eqs. (14) and (15) we find that for the initial beam generated at the output of the MZS the beam quality and kurtosis parameters, normalized to Gaussian beam values $\left(Q_{g}\right.$ and $\left.K_{g}\right)$ with the same wavelength, take the values $Q / Q_{g}=8.02$ and $K / K_{g}=2.02$, respectively. Moreover, by using Eqs. (2), (3), and (8) the beam quality $Q^{\prime} / Q_{g}$ and the kurtosis parameter $K^{\prime} / K_{g}$ at the output of the polarizer for different angles $\theta$ can be computed. The results have been represented in Figs. 2 and 3. From Fig. 2 we see that the beam quality for $\theta=0^{\circ}$ takes the value $Q_{0^{\circ}}{ }^{\prime} / Q_{g}$ $=6.43$ and for $\theta=90^{\circ}, Q_{90^{\circ}} / Q_{g}=1.394$. The minimum value of $Q^{\prime} / Q_{g}$ (best quality) is reached for $\theta=88.81^{\circ}\left(Q^{\prime} / Q_{g}=1.390\right)$, and the maximum $\left(Q^{\prime} / Q_{g}=190.178\right)$, for $\theta=134.65^{\circ}$. For the kurtosis parameter in Fig. 3 we see that $K^{\prime} / K_{g}$ takes values from 0.61 to 2.17 . For $\theta=0^{\circ}$ and $\theta=90^{\circ}$ the kurtosis takes the values $K_{0^{\circ}}{ }^{\prime} / K_{g}=0.61$ and $K_{90^{\circ}}{ }^{\prime} / K_{g}=1.58$, respectively. For the angles $\theta=10.31^{\circ}, 111.15^{\circ}$, $142.95^{\circ}$, and $171.42^{\circ}$ we have a mesokurtic beam $\left(K^{\prime} / K_{g}=1\right)$. For the rest of the angles the beam changes from leptokurtic to platykurtic and vice versa.

From the above we see that it is possible to obtain beams with different global parameters in a simple way by rotating the transmission axis of the polarizer. On the other hand, diffraction effects are expected to be nonnoticeable because we handle softedge apertures. Finally, we would like to remark that the values of the global parameters can be changed at will in the proposed system by modifying the characteristics of the transmittances.

\section{Conclusions}

A method has been proposed for global beam shaping that modifies two characteristic parameters of a beam: the beam quality parameter and the kurtosis parameter. The procedure is based on the synthesis of a totally and nonuniformly polarized beam using a Mach-Zehnder system with orthogonal polarizers, different amplitude transmittances at each arm, and a linear polarizer at the output of the interferometer.
By rotating this last polarizer a continuous change of the global parameters is obtained.

This work was supported by the Ministerio de Educación y Ciencia of Spain, under project FIS2004-1900 and by the Universidad Complutense-Comunidad de Madrid, within the framework of the Research Groups Program 2005-06. The authors thank J. Serna for his valuable suggestions in the course of reading the manuscript.

\section{References}

1. P. W. Rhodes and D. L. Shealy, "Refractive optical systems for irradiance redistribution of collimated radiation: their design and analysis," Appl. Opt. 19, 3545-3553 (1980).

2. D. G. Burkhard and D. L. Shealy, "Simplified formula for the illuminance in an optical system," Appl. Opt. 20, 897-909 (1981).

3. K. Iwasaki, T. Hayashi, T. Goto, and S. Shimizu, "Square and uniform laser output device: theory and applications," Appl. Opt. 90, 1736-1744 (1990).

4. G. Piquero, P. M. Mejías, and R. Martínez-Herrero, "Linear Gaussian intensity distributions synthesized by reflection on elliptic cylinders: a proposal," Appl. Opt. 31, 2970-2971 (1992).

5. W. B. Veldkamp, "Laser beam profile shaping with interlaced binary diffraction gratings," Appl. Opt. 21, 3209-3212 (1982).

6. C. S. Ih, "Absorption lens for producing uniform laser beam," Appl. Opt. 11, 694-695 (1972).

7. L. W. Casperson, N. K. Kincheloe, and O. M. Stafsudd, "Phase plates for laser beam compensation," Opt. Commun. 21, 1-4 (1977).

8. W.-H. Lee, "Method for converting a Gaussian beam into a uniform beam," Opt. Commun. 36, 469-471 (1981).

9. W. W. Simmons, G. W. Leppelmeier, and B. C. Johnson, "Optical beam shaping devices using polarization effects," Appl. Opt. 13, 1629-1632 (1974).

10. B. J. Feldman and S. J. Gitomer, "Anular lens soft aperture for high-power laser systems,” Appl. Opt. 15, 1379-1380 (1976).

11. G. Giuliani, Y. K. Park, and R. L. Byer, "Radial birefringent element and its application to laser resonator design," Opt. Lett. 5, 491-493 (1980).

12. S. Lavi, R. Prochaska, and E. Keren, "Generalized beam parameters and transformation law for partially coherent light," Appl. Opt. 27, 3696-3703 (1988).

13. M. J. Bastiaans, "Propagation laws for the second-order moments of the Wigner distribution function in first-order optical systems," Optik 82, 173-181 (1989).

14. A. E. Siegman, "New developments in laser resonators," in Proc. SPIE 1224, 2-14 (1990).

15. J. Serna, R. Martínez-Herrero, and P. M. Mejías, "Parametric characterization of general partially coherent beams propagating through $A B C D$ optical systems," J. Opt. Soc. Am. A 8, 1094-1098 (1991).

16. H. Weber, "Propagation of higher-order intensity moments in quadratic-index media," Opt. Quantum Electron. 24, 10271049 (1992).

17. G. Piquero, P. M. Mejías, and R. Martínez-Herrero, "On the kurtosis parameter of laser beams," in Proceedings of the First Workshop on Laser Beam Characterization, P. M. Mejías, H. Weber, R. Martínez-Herrero, and A. González Ureña, eds., (Sociedad Española de Óptica, 1993), pp. 141-148.

18. A. E. Siegman, "Binary phase plates cannot improve laser beam quality," Opt. Lett. 18, 675-677 (1993).

19. R. Martínez-Herrero, P. M. Mejías, and G. Piquero, "Quality improvement of partially coherent symmetric-intensity beams caused by quartic phase distorsions," Opt. Lett. 17, 1650-1651 (1992). 
20. G. Piquero, P. M. Mejías, and R. Martínez-Herrero, "Sharpness changes of Gaussian beams induced by spherically aberrated lenses," Opt. Commun. 107, 179-83 (1994).

21. L. Shirong and B. Lu, "M2 factor and kurtosis parameter of super-Gaussian beams passing through an axicon," Optik 114, 193-198 (2003).
22. Q. Lü, S. Dong, and H. Weber, "Analysis of $T E M_{00}$ laser beam degradation caused by a birefringent Nd:YAG rod," Opt. Quantum Electron. 27, 777-783 (1995).

23. V. G. Niziev and A. V. Nesterov, "Influence of beam polarization on laser cutting efficiency," J. Phys. D. 32, 1455-1461 (1999). 